\title{
Correction to: Demographic, Socioeconomic, Health Systems, and Geographic Factors Associated with Vaginal Birth After Cesarean: An Analysis of 2017 U.S. Birth Certificate Data
}

\author{
Bridget Basile Ibrahim ${ }^{1}\left[\right.$ [ $\cdot$ Holly Powell Kennedy ${ }^{1} \cdot$ Margaret L. Holland $^{1}$
}

Published online: 7 April 2021

๑) Springer Science+Business Media, LLC, part of Springer Nature 2021

\section{Correction to: Maternal and Child Health Journal https://doi.org/10.1007/s10995-020-03066-3}

The authors would like to correct the errors in the publication of the original article. The corrected details are given below.

(a) Page 2: "In spite of high success rates (Cunningham et al. 2010) and strong recommendations from birth professional organizations and the National Institutes of Health (American College of Nurse-Midwives 2017; American College of Obstetricians and Gynecologists 2019; Cunningham et al. 2010) (Attanasio et al. 2019; Declercq et al. 2002, 2006, 2014), rates of VBAC in the United States have hovered just above $10 \%$ of births following cesarean for over ten years (Martin et al. 2018). This is despite fact that consistently over the past decade, approximately half of women with a history of cesarean were interested in having a VBAC (Declercq et al. 2002, 2006, 2014)." should be changed to "In spite of high success rates
(Cunningham et al. 2010) and strong recommendations from birth professional organizations and the National Institutes of Health (American College of Nurse-Midwives 2017; American College of Obstetricians and Gynecologists 2019; Cunningham et al. 2010) rates of VBAC in the United States have hovered just above $10 \%$ of births following cesarean for over ten years (Martin et al. 2018). This is despite the fact that consistently over the past decade, approximately half of women with a history of cesarean were interested in having a VBAC (Attanasio et al. 2019; Declercq et al. 2002, 2006, 2014)."

(b) Page 7: The odds ratio $(a \mathrm{OR})$ and confidence intervals ( $95 \% \mathrm{CI})$ in last portion of Table 3 referring to "Geographic region" were errantly printed in the incorrect location. These figures were shifted to the right to place them in the correct column entitled "Block 4: geography". A corrected version of the table appears below.

The original article has been corrected.

The original article can be found online at https://doi.org/10.1007/ s10995-020-03066-3.

Bridget Basile Ibrahim

bridget.basileibrahim@yale.edu

1 Yale University School of Nursing, 400 West Campus Drive, Orange, CT 06477, USA 
Table 3 Block logistic regression of sociodemographic, systems, and geographic factors associated with VBAC birth in the United States in 2017 (N = 540,711) (from 2017 U.S. Birth Certificate Data)

\begin{tabular}{|c|c|c|c|c|c|c|c|c|c|c|c|c|}
\hline \multirow{3}{*}{$\begin{array}{l}\text { C statistic } \\
\text { Variable }\end{array}$} & \multicolumn{3}{|c|}{ Block 1: demographic } & \multicolumn{3}{|c|}{ Block 2: socioeconomic } & \multicolumn{3}{|c|}{ Block 3: system factors } & \multicolumn{3}{|c|}{ Block 4: geography } \\
\hline & \multicolumn{3}{|c|}{0.512} & \multicolumn{3}{|l|}{0.547} & \multicolumn{3}{|c|}{0.562} & \multicolumn{3}{|l|}{0.583} \\
\hline & $a \mathrm{OR}$ & $95 \% \mathrm{Cl}$ & & $a \mathrm{OR}$ & $95 \% \mathrm{CI}$ & & $a \mathrm{OR}$ & $95 \% \mathrm{CI}$ & & $a \mathrm{OR}$ & $95 \% \mathrm{Cl}$ & \\
\hline \multicolumn{13}{|l|}{ Maternal age } \\
\hline Per +5 year increment & 0.98 & 0.97 & 0.98 & 0.97 & 0.96 & 0.97 & 0.96 & 0.95 & 0.96 & 0.95 & 0.94 & 0.96 \\
\hline Race & \multicolumn{12}{|c|}{$($ Ref = White $)$} \\
\hline Black & 1.03 & 1.01 & 1.05 & 1.03 & 1.01 & 1.05 & 0.97 & 0.95 & 0.99 & 1.06 & 1.04 & 1.08 \\
\hline American Indian-Alaska Native & 1.18 & 1.10 & 1.27 & 1.17 & 1.09 & 1.26 & 1.23 & 1.15 & 1.33 & 1.18 & 1.10 & 1.27 \\
\hline Asian & 1.03 & 1.003 & 1.06 & 0.97 & 0.95 & 1.01 & 0.91 & 0.89 & 0.94 & 0.91 & 0.88 & 0.94 \\
\hline Ethnicity & \multicolumn{12}{|c|}{$($ Ref $=$ not Latina $)$} \\
\hline Latina & 0.98 & 0.96 & 1.001 & 0.91 & 0.89 & 0.93 & 0.86 & 0.84 & 0.88 & 0.90 & 0.88 & 0.92 \\
\hline Maternal education & \multicolumn{12}{|c|}{$($ Ref $=4$ year degree or greater $)$} \\
\hline No high school & & & & 1.13 & 1.09 & 1.16 & 1.15 & 1.12 & 1.19 & 1.13 & 1.10 & 1.16 \\
\hline High school/GED & & & & 0.84 & 0.82 & 0.86 & 0.86 & 0.84 & 0.88 & 0.85 & 0.83 & 0.88 \\
\hline Some college & & & & 0.84 & 0.83 & 0.86 & 0.86 & 0.85 & 0.89 & 0.85 & 0.84 & 0.87 \\
\hline Payor & \multicolumn{12}{|c|}{ (Ref = private insurance $)$} \\
\hline Medicaid & & & & 1.03 & 1.01 & 1.05 & 1.04 & 1.02 & 1.38 & 1.05 & 1.03 & 1.07 \\
\hline Self-pay & & & & 1.76 & 1.69 & 1.83 & 1.86 & 1.79 & 1.93 & 1.99 & 1.92 & 2.07 \\
\hline Residence county population & \multicolumn{12}{|c|}{$(\operatorname{Ref}=<100 \mathrm{k}$ persons $)$} \\
\hline $1,000,000+$ & & & & & & & 1.31 & 1.27 & 1.34 & 1.21 & 1.18 & 1.24 \\
\hline $500,000-1,000,000$ & & & & & & & 1.51 & 1.47 & 1.54 & 1.43 & 1.40 & 1.47 \\
\hline $250,000-500,00$ & & & & & & & 1.39 & 1.35 & 1.43 & 1.36 & 1.32 & 1.40 \\
\hline $100,000-250,000$ & & & & & & & 1.25 & 1.21 & 1.28 & 1.23 & 1.19 & 1.26 \\
\hline Geographic region & \multicolumn{12}{|c|}{$($ Ref $=$ Northeast $)$} \\
\hline South & & & & & & & & & & 0.72 & 0.71 & 0.74 \\
\hline Midwest & & & & & & & & & & 1.19 & 1.16 & 1.22 \\
\hline West & & & & & & & & & & 1.06 & 1.03 & 1.08 \\
\hline
\end{tabular}

Publisher's Note Springer Nature remains neutral with regard to jurisdictional claims in published maps and institutional affiliations. 